Bull. Korean Math. Soc. 51 (2014), No. 5, pp. 1503-1510

http://dx.doi.org/10.4134/BKMS.2014.51.5.1503

\title{
PERSISTENCE OF HOMOCLINIC ORBITS AFTER DISCRETIZATION OF A TWO DIMENSIONAL DEGENERATE DIFFERENTIAL SYSTEM
}

\author{
Noureddine Mehidi And Nadia Mohdeb
}

\begin{abstract}
The aim of this work is to construct a general family of two dimensional differential systems which admits homoclinic solutions near a non-hyperbolic fixed point, such that a Jacobian matrix at this point is zero. We then discretize it by using Euler's method and look after the persistence of the homoclinic solutions in the obtained discrete system.
\end{abstract}

\section{Introduction}

Consider the vector field

$$
\left\{\begin{array}{l}
\dot{x}=F(x, y) \\
\dot{y}=G(x, y)
\end{array}\right.
$$

where $(\cdot)=d / d t$ and $F$ and $G$ are functions of class $\mathcal{C}^{1}$ from $\mathbb{R}^{2}$ into $\mathbb{R}$. We suppose that the origin is an isolated equilibrium point of system (1) and that the Jacobian matrix at this point has two real eigenvalues $\lambda_{1}$ and $\lambda_{2}$. In the case where only $\lambda_{1}$ is null, the origin is a node, a saddle or a point with one parabolic and two hyperbolic sectors [12]. When $\lambda_{1}=\lambda_{2}=0$, the origin is non-hyperbolic and the situation is more complicated; In the case where the matrix of the linear part of system (1) is not zero, a local description of system (1) has been studied in [1].

In the case of non-degenerate homoclinic orbits of an autonomous equation, references ([2], [10], [14]) give an answer to the question of persistence of homoclinic solutions in the discretized system. The problems of numerical computation of homoclinic and heteroclinic orbits and that of approximation of phase portraits are studied in ([3]-[10], [14]).

Knowing that after discretization by the Euler's method the solution pulls outwards, contrary to the idea which consists in believing that homoclinic solutions do not persist in the associated discretized system, this work aims to

Received August 1, 2013

2010 Mathematics Subject Classification. 34C37, 34A34, 39A05.

Key words and phrases. homoclinic orbits, degenerate system, non-hyperbolic fixed point, discrete system. 
show that in degenerate systems, this is not necessarily the case. Thus, we consider a general family of differential equations with zero linear part, and homoclinic orbits surrounding the fixed point, and we study the persistence of these solutions after Euler discretization. We then consider the differential system

$$
\left\{\begin{array}{l}
\dot{x}=\left(y-\varphi_{1}(x)\right)\left(\varphi_{2}(x)-y\right) f(x, y) \\
\dot{y}=x(y-\psi(x)) g(x)
\end{array}\right.
$$

where $f$ is of class $\mathcal{C}^{1}$ from $\mathbb{R}^{2}$ into $\mathbb{R}, g, \varphi_{1}, \varphi_{2}$ and $\psi$ are of class $\mathcal{C}^{1}$ from $\mathbb{R}$ into $\mathbb{R}$ and $\varphi_{1}, \varphi_{2}$ and $\psi$ are surjective. We have already study this problem [13] in the case of the differential system corresponding to the iteration of the quadratic complex polynomial $z^{2}+c$. In all what follows, we assume that the origin is the unique singular point of system (2) and,

i) $\forall x, y \in \mathbb{R}, f(x, y)>0, g(x)>0$.

ii) $\varphi_{1}(0)=\varphi_{2}(0)=\psi(0)=0$.

iii) $\forall x \in \mathbb{R}, \varphi_{1}^{\prime}(x)>0, \varphi_{2}^{\prime}(x)<0, \psi^{\prime}(x)>0$.

iv) $\forall x>0, \varphi_{1}(x)>\psi(x)>\varphi_{2}(x)$ and $\forall x<0, \varphi_{1}(x)<\psi(x)<\varphi_{2}(x)$.

In Section 2, we prove that all trajectories of system (2) which start from the region $R=\left\{(x, y) \in \mathbb{R}^{2} ; y>\varphi_{1}(x), y>\varphi_{2}(x), x<0\right\}$ are homoclinic. In Section 3, we prove that for a $r_{0}>0$, solutions passing through the set

$$
\bar{R}=\left\{(x, y) \in \mathbb{R}^{2} ; x^{2}+y^{2} \leq r_{0}^{2}, y>\varphi_{1}(x), y>\varphi_{2}(x), x<0\right\}
$$

remain homoclinic in the discrete system obtained by Euler discretization.

\section{Homoclinic orbits in the continuous system}

Under hypotheses (i)-(iv), the following proposition provides the existence of homoclinic solutions in system (2).

Proposition 1. The orbits of system (2) starting from region $R$ are homoclinic.

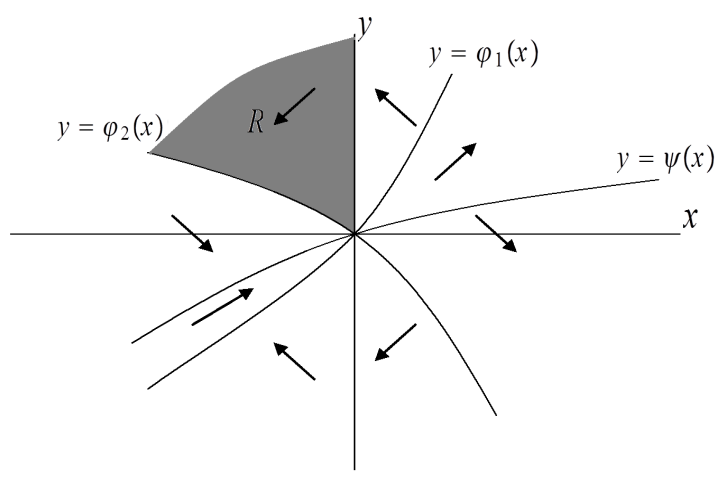

Figure 1. Vector field (2). 
Proof. Let $\gamma_{0}=(x(t), y(t))$ be the trajectory of system (2) starting from a point $\left(x_{0}, y_{0}\right)$ of $R$.

The trajectory $\gamma_{0}$ crosses the curve $y=\varphi_{2}(x)$ and enters the region

$$
\left\{(x, y) \in \mathbb{R}^{2} ; y>\varphi_{1}(x), y<\varphi_{2}(x)\right\}
$$

which is invariant under system (2) (Figure 1). In this region $x(t)$ is increasing and upper bounded. Besides $y(t)$ is bounded and increasing or decreasing following the fact that it crosses the curve $y=\psi(x)$ or not. Hence, $\gamma_{0}$ tends toward $(0,0)$ whenever $t \rightarrow+\infty$.

In the same way, it can be proved that $\gamma_{0}$ tends toward $(0,0)$ whenever $t \rightarrow-\infty$.

Remark 1. The case where the orbits of system (2) are started from the region is similar.

$$
\left\{(x, y) \in \mathbb{R}^{2} ; y<\varphi_{1}(x), y<\varphi_{2}(x), x<0\right\}
$$

\section{Homoclinic orbits in the discretized system}

A discretization of (2) by the Euler's method gives

$$
\left\{\begin{array}{l}
x_{n+1}=x_{n}+h\left(y_{n}-\varphi_{1}\left(x_{n}\right)\right)\left(\varphi_{2}\left(x_{n}\right)-y_{n}\right) f\left(x_{n}, y_{n}\right) \\
y_{n+1}=y_{n}+h x_{n}\left(y_{n}-\psi\left(x_{n}\right)\right) g\left(x_{n}\right)
\end{array}\right.
$$

where $h>0$ is the stepsize of the discretization.

In all what follows, $\left(x_{0}, y_{0}\right)$ is a point of the region $\left\{(x, y) \in \mathbb{R}^{2} ; y>\psi(x)\right\}$. Suppose that the assumptions (i)-(iv) above are satisfied for system (3). We have the following lemmas:

Lemma 1. There exist $a h_{0}>0$ and $a r_{0}>0$ such that for any $h \in\left(0, h_{0}\right]$, any point $\left(x_{0}, y_{0}\right)$ in the ball $\mathcal{B}_{r_{0}}=\left\{(x, y) \in \mathbb{R}^{2} ; x^{2}+y^{2} \leq r_{0}^{2}\right\}$ has a unique predecessor in $\mathcal{B}_{r_{0}+1}$.

Proof. We have

$$
\begin{aligned}
x_{n+1}= & x_{n}+h\left(\frac{y_{n+1}+h \psi\left(x_{n}\right) g\left(x_{n}\right)}{1+h g\left(x_{n}\right)}-\varphi_{1}\left(x_{n}\right)\right) \\
& \times\left(\varphi_{2}\left(x_{n}\right)-\frac{y_{n+1}+h \psi\left(x_{n}\right) g\left(x_{n}\right)}{1+h g\left(x_{n}\right)}\right) f\left(x_{n}, \frac{y_{n+1}+h \psi\left(x_{n}\right) g\left(x_{n}\right)}{1+h g\left(x_{n}\right)}\right) .
\end{aligned}
$$

Let us denote for a given $\left(x_{n+1}, y_{n+1}\right)$,

$$
\begin{aligned}
\mathcal{F}(x)= & x+h\left(\frac{y_{n+1}+h \psi(x) g(x)}{1+h g(x)}-\varphi_{1}(x)\right) \\
& \times\left(\varphi_{2}(x)-\frac{y_{n+1}+h \psi(x) g(x)}{1+h g(x)}\right) f\left(x, \frac{y_{n+1}+h \psi(x) g(x)}{1+h g(x)}\right)-x_{n+1} .
\end{aligned}
$$

There exist a $h_{0}>0$ and a $r_{0}>0$ such that for any $h \in\left(0, h_{0}\right]$ and for $\left(x_{n+1}, y_{n+1}\right)$ in $\mathcal{B}_{r_{0}}$,

$$
\mathcal{F}\left(x_{n+1}-1\right)<0
$$


and

$$
\mathcal{F}\left(x_{n+1}+1\right)>0 .
$$

Moreover, for any $x$ in $] x_{n+1}-1, x_{n+1}+1\left[, \mathcal{F}^{\prime}(x)>0\right.$. As $\mathcal{F}$ is continuous, equation $\mathcal{F}(x)=0$ admits a unique solution $x$ in $] x_{n+1}-1, x_{n+1}+1\left[\right.$. For $x_{n}$ in $] x_{n+1}-1, x_{n+1}+1\left[\right.$, we get $y_{n}=\left(y_{n+1}+h x_{n} \psi\left(x_{n}\right) g\left(x_{n}\right)\right) /\left(1-h x_{n}\right) \in$ ]$y_{n+1}-1, y_{n+1}+1[$.

Lemma 2. There exist $a h_{0}>0$ and $a r_{0}>0$ such that for any $h \in$ $\left(0, h_{0}\right]$ and any $\left(x_{0}, y_{0}\right)$ in the set $\left\{(x, y) \in \mathbb{R}^{2} ; x^{2}+y^{2} \leq r_{0}^{2}, y>\varphi_{1}(x)\right\}$, the solution $\left(x_{n}, y_{n}\right)_{n \in \mathbb{N}}$ of system (3) through $\left(x_{0}, y_{0}\right)$ does not enter the region $\left\{(x, y) \in \mathbb{R}^{2} ; y<\varphi_{1}(x)\right\}$.

Proof. For $x_{n}<0$,

$$
\begin{aligned}
& y_{n+1}-\varphi_{1}\left(x_{n+1}\right) \\
\geq & \left(y_{n}-\varphi_{1}\left(x_{n}\right)\right)\left(1+h x_{n} g\left(x_{n}\right)-h\left(\varphi_{2}\left(x_{n}\right)-y_{n}\right) f\left(x_{n}, y_{n}\right) \varphi_{1}^{\prime}(c)\right)
\end{aligned}
$$

for some $c$ in $] x_{n}, x_{n+1}$ [ if $x_{n}<x_{n+1}$ or $c$ in $] x_{n+1}, x_{n}\left[\right.$ if $x_{n}>x_{n+1}$. Hence, there exist a $h_{0}>0$ and a $r_{0}>0$ such that for any $h \in\left(0, h_{0}\right]$ and for $x_{0}^{2}+y_{0}^{2} \leq r_{0}^{2}$, we get by induction

$$
y_{n+1}-\varphi_{1}\left(x_{n+1}\right)>0, \forall n \in \mathbb{N}
$$

whenever $y_{0}-\varphi_{1}\left(x_{0}\right)>0$.

Lemma 3. There exist $a h_{0}>0$ and $a r_{0}>0$ such that for any $h \in\left(0, h_{0}\right]$ and for any $\left(x_{0}, y_{0}\right)$ in the set $\left\{(x, y) \in \mathbb{R}^{2} ; \varphi_{1}(x)<y<\psi(x)\right\}$ with $x_{0}^{2}+y_{0}^{2} \leq r_{0}^{2}$, the solution $\left(x_{n}, y_{n}\right)_{n \in \mathbb{N}}$ of system (3) through $\left(x_{0}, y_{0}\right)$ does not leave this set.

Proof. We have,

$$
y_{n+1}-\psi\left(x_{n+1}\right)=\left(y_{n}-\psi\left(x_{n}\right)\right)\left(1+h x_{n} g\left(x_{n}\right)\right)+\left(x_{n}-x_{n+1}\right) \psi^{\prime}(d),
$$

where $d \in] x_{n}, x_{n+1}\left[\right.$ if $x_{n}<x_{n+1}$ and $\left.d \in\right] x_{n+1}, x_{n}\left[\right.$ if $x_{n}>x_{n+1}$. Hence,

$$
\begin{aligned}
& y_{n+1}-\psi\left(x_{n+1}\right) \\
\leq & \left(y_{n}-\psi\left(x_{n}\right)\right)\left(1+h\left(x_{n} g\left(x_{n}\right)-\left(\varphi_{2}\left(x_{n}\right)-y_{n}\right) f\left(x_{n}, y_{n}\right) \psi^{\prime}(d)\right)\right) .
\end{aligned}
$$

Using Lemma 2, there exist a $h_{0}>0$ and a $r_{0}>0$ such that for any $h \in\left(0, h_{0}\right]$ and for $x_{0}^{2}+y_{0}^{2} \leq r_{0}^{2}, y_{n+1}-\psi\left(x_{n+1}\right)<0$ if $y_{0}<\psi\left(x_{0}\right)$.

Lemma 4. For any point $\left(x_{0}, y_{0}\right)$ in $R$, there exists $n_{2}$ in $\mathbb{N}^{*}$ such that $y_{n_{2}}<$ $\varphi_{2}\left(x_{n_{2}}\right)$.

Proof. Suppose that the solution $\left(x_{n}, y_{n}\right)_{n \in \mathbb{N}}$ of system (3) started from $\left(x_{0}, y_{0}\right)$ does not leave the region

$$
\left\{(x, y) \in \mathbb{R}^{2} ; x<0, y>\varphi_{2}(x)\right\} .
$$

The sequences $\left(x_{n}\right)_{n \in \mathbb{N}}$ and $\left(y_{n}\right)_{n \in \mathbb{N}}$ are decreasing in this region. Thus, the sequence $\left(x_{n}\right)_{n \in \mathbb{N}}$ is down bounded. It converges toward a limit $l<x_{0}$. Moreover, following Lemma 2, the sequence $\left(y_{n}\right)_{n \in \mathbb{N}}$ is down bounded, thus converges. 
A passage to the limit implies that the limit of the sequence $\left(x_{n}, y_{n}\right)_{n \in \mathbb{N}}$ is the unique stationary point $(0,0)$ of system $(2)$. There is a contradiction with the fact that $l<0$.

Lemma 5. There exist a $h_{0}>0$ and a $r_{0}>0$ such that for any $h \in\left(0, h_{0}\right]$ and for any $\left(x_{0}, y_{0}\right)$ in the set

$$
\left\{(x, y) \in \mathbb{R}^{2} ; x^{2}+y^{2} \leq r_{0}^{2}, x<0, \varphi_{1}(x)<y<\varphi_{2}(x)\right\},
$$

the solution $\left(x_{n}, y_{n}\right)_{n \in \mathbb{N}}$ of system (3) through $\left(x_{0}, y_{0}\right)$ does not enter the region $\left\{(x, y) \in \mathbb{R}^{2} ; x<0, y>\varphi_{2}(x)\right\}$.

Proof. There is a $c \in] x_{n}, x_{n+1}[$ such that

$$
\begin{aligned}
\varphi_{2}\left(x_{n+1}\right)-y_{n+1}= & \left(\varphi_{2}\left(x_{n}\right)-y_{n}\right)\left(h\left(y_{n}-\varphi_{1}\left(x_{n}\right)\right) f\left(x_{n}, y_{n}\right) \varphi_{2}^{\prime}(c)+1\right) \\
& -h x_{n}\left(y_{n}-\psi\left(x_{n}\right)\right) g\left(x_{n}\right) .
\end{aligned}
$$

It follows that, for $x_{n}<0$,

$$
\begin{aligned}
& \varphi_{2}\left(x_{n+1}\right)-y_{n+1} \\
\geq & \left(\varphi_{2}\left(x_{n}\right)-y_{n}\right)\left(1+h\left(y_{n}-\varphi_{1}\left(x_{n}\right)\right) f\left(x_{n}, y_{n}\right) \varphi_{2}^{\prime}(c)-h x_{n} g\left(x_{n}\right)\right) .
\end{aligned}
$$

Thus, there exist a $h_{0}>0$ and a $r_{0}>0$ such that for any $h \in\left(0, h_{0}\right]$ and for $x_{0}^{2}+y_{0}^{2} \leq r_{0}^{2}$,

since $\varphi_{2}\left(x_{0}\right)>y_{0}$.

$$
\varphi_{2}\left(x_{n+1}\right)-y_{n+1}>0
$$

Given a point $\left(x_{0}, y_{0}\right)$, according to Lemma 1 , let be the solution

$$
\left(x_{-n}, y_{-n}\right)_{n \in \mathbb{N}}
$$

of system

$$
\left\{\begin{aligned}
x_{-n}=x_{-n-1}+ & h\left(y_{-n-1}-\varphi_{1}\left(x_{-n-1}\right)\right)\left(\varphi_{2}\left(x_{-n-1}\right)-y_{-n-1}\right) \\
& \times f\left(x_{-n-1}, y_{-n-1}\right) \\
y_{-n}=y_{-n-1}+ & h\left(y_{-n-1}-\psi\left(x_{-n-1}\right)\right) x_{-n-1} g\left(x_{-n-1}\right)
\end{aligned}\right.
$$

satisfying $\left(x_{-1}, y_{-1}\right) \simeq\left(x_{0}, y_{0}\right)$ and the condition that there exist a $h_{0}>0$ and a $r_{0}>0$ such that

$$
\left(x_{-n-1}, y_{-n-1}\right) \simeq\left(x_{-n}, y_{-n}\right)
$$

whenever $\left(x_{-n}, y_{-n}\right) \leq r_{0}^{2}$.

Lemma 6. There exist $a h_{0}>0$ and $a r_{0}>0$ such that for any $h \in\left(0, h_{0}\right]$ and for any $\left(x_{0}, y_{0}\right)$ in $\bar{R}$, i)

Moreover,

$$
\forall n \in \mathbb{N}, y_{-n}>\psi\left(x_{-n}\right) .
$$

ii)

$$
\exists k \in \mathbb{N}^{*}, y_{-k}<\varphi_{1}\left(x_{-k}\right)
$$

$$
\forall n>k, y_{-n}<\varphi_{1}\left(x_{-n}\right)
$$


Proof. i) Suppose that

$$
\exists p \in \mathbb{N}^{*}, y_{-p}<\psi\left(x_{-p}\right) .
$$

It follows that the sequence $\left(y_{-n}\right)_{-n>-p}$ of successors of $y_{-p}$ satisfies $y_{-n}<$ $\psi\left(x_{-n}\right)$ (because it can be proved by induction that if $y_{0}<\psi\left(x_{0}\right)$, then $y_{n}<\psi\left(x_{n}\right)$ for all $n$ in $\left.\mathbb{N}^{*}\right)$. It follows in particular that $y_{0}<\psi\left(x_{0}\right)$. This is a contradiction.

In order to prove the second part of (i), suppose that the solution

$$
\left(x_{-n}, y_{-n}\right)_{n \in \mathbb{N}}
$$

does not leave the region $\left\{(x, y) \in \mathbb{R}^{2} ; y>\varphi_{1}(x), x>0\right\}$.

The sequence $\left(x_{-n}\right)_{n \in \mathbb{N}}$ is increasing and $\left(y_{-n}\right)_{n \in \mathbb{N}}$ is decreasing in this region. The sequence $\left(x_{-n}\right)_{n \in \mathbb{N}}$ is upper bounded. It converges toward a limit $l>x_{0}$. Moreover, the sequence $\left(y_{-n}\right)_{n \in \mathbb{N}}$ is down bounded, and then converges. The limit of the sequence $\left(x_{-n}, y_{-n}\right)_{n \in \mathbb{N}}$ is $(0,0)$. There is a contradiction with the fact that $l$ is strictly positive.

ii) Suppose that there is $q$ in $\mathbb{N}^{*}, q>k$ such that $y_{-q}>\varphi_{1}\left(x_{-q}\right)$. It follows that for any $n$ in $\mathbb{N}^{*}$ such that $n<q$, we have $y_{-n}>\varphi_{1}\left(x_{-n}\right)$. In particular, $y_{-k}>\varphi_{1}\left(x_{-k}\right)$. This is a contradiction.

We can now give and prove the main result:

Theorem. There exist $a h_{0}>0$ and $a r_{0}>0$ such that for any $h \in\left(0, h_{0}\right]$ and for any point $\left(x_{0}, y_{0}\right)$ in $\bar{R}$, the solution $\left(x_{n}, y_{n}\right)_{n \in \mathbb{N}}$ of system (3) emanating from $\left(x_{0}, y_{0}\right)$ is homoclinic to the origin.

Proof. By using Lemma 4, the solution $\left(x_{n}, y_{n}\right)_{n \in \mathbb{N}}$ of system (3) started from point $\left(x_{0}, y_{0}\right)$ enters the region $\left\{(x, y) \in \mathbb{R}^{2} ; \psi(x)<y<\varphi_{2}(x)\right\}$ and,

- either the solution $\left(x_{n}, y_{n}\right)_{n \in \mathbb{N}}$ remains in this region, then the sequence $\left(x_{n}\right)_{n \in \mathbb{N}}$ is increasing and upper bounded while the sequence $\left(y_{n}\right)_{n \in \mathbb{N}}$ is decreasing and down bounded,

- or the solution $\left(x_{n}, y_{n}\right)_{n \in \mathbb{N}}$ enters the region $\left\{(x, y) \in \mathbb{R}^{2} ; \varphi_{1}(x)<y<\psi(x)\right\}$, then by Lemmas 2, 3 and 5 , it does not leave it. Hence, the sequences $\left(x_{n}\right)_{n \in \mathbb{N}}$ and $\left(y_{n}\right)_{n \in \mathbb{N}}$ are increasing and upper bounded.

Consequently, in the two cases, the sequence $\left(x_{n}, y_{n}\right)_{n \in \mathbb{N}}$ is convergent. Its limit is $(0,0)$, the unique stationary point of $(3)$.

In the same way using Lemma 6 , it can be proved that the sequence

$$
\left(x_{-n}, y_{-n}\right)_{n \in \mathbb{N}}
$$

which is well defined according to Lemma 1 , converges toward $(0,0)$ when $n$ tends toward $+\infty$.

Remark 2. The same work can be realized if we take

$$
\dot{y}=(y-\psi(x))\left(y-\psi_{1}(x)\right) g(x)
$$

instead of

$$
\dot{y}=x(y-\psi(x)) g(x),
$$


where $\psi_{1}$ is a $\mathcal{C}^{1}$ and strictly increasing function such that $\psi_{1}(0)=0$ and,

$$
\left\{\begin{array}{l}
\varphi_{1}(x)<\psi_{1}(x)<\varphi_{2}(x) \text { if } x>0 \\
\varphi_{1}(x)>\psi_{1}(x)>\varphi_{2}(x) \text { if } x<0
\end{array}\right.
$$

Remark 3. It can be proved in the same way that there exist a $h_{0}>0$ and a $r_{1}>0$ such that for any $h \in\left(0, h_{0}\right]$ the solutions of systems $(2)$ and $(3)$ started from the region $\left\{(x, y) \in \mathbb{R}^{2} ; x<0, x^{2}+y^{2} \leq r_{1}^{2}, y<\varphi_{1}(x), y<\varphi_{2}(x)\right\}$, are homoclinics.

\section{References}

[1] A. A. Andronov, E. A. Leontovich, I. I. Gordon, and A. G. Maier, Qualitative Theory of Second Order Dynamic Systems, John Wiley and Sons, New York, Toronto, 1973.

[2] W. J. Beyn, The effect of discretization on homoclinic orbits, Bifurcation, Analysis, Algorithms, Applications, Birkhäuser, Basel, 1-8, 1987.

[3] _ On invariant closed curves for one-step methods, Numer. Math. 51 (1987), no. $1,103-122$.

[4] W. J. Beyn and B. M. Garay, Estimates of variable stepsize Runge-Kutta methods for sectorial evolution equations with nonsmooth data, Appl. Numer. Math. 41 (2002), no. 3, 369-400.

[5] W. J. Beyn and J. M. Kleinhauf, The numerical computation of homoclinic orbits for maps, SIAM J. Numer. Anal. 34 (1997), no. 3, 1207-1236.

[6] W. J. Beyn and M. Stiefenhofer, A direct approach to homoclinic orbits in the fast dynamics of singularly perturbed systems, J. Dynam. Differential Equations 11 (1999), no. 4, 671-709.

[7] N. Chen, J. Sun, Y. Sun, and M. Tang, Visualizing the complex dynamics of families of polynomials with symmetric critical points, Chaos Solitons Fractals 42 (2009), no. 3, 1611-1622.

[8] E. J. Doedel, M. J. Friedman, and B. I. Kunin, Successive continuation for locating connecting orbits, Numer. Algorithms 14 (1997), no. 1-3, 103-124.

[9] E. J. Doedel, M. J. Friedman, and A. C. Monteiro, On locating connecting orbits, Appl. Math. Comput. 65 (1994), no. 1-3, 231-239.

[10] B. Fiedler and J. Sheurle, Discretization of homoclinic orbits, rapid forcing and "invisible" chaos, Mem. Amer. Math. Soc. 119 (1996), no. 570, viii+79 pp.

[11] S. J. Greenfield and R. D. Nussbaum, Dynamics of a quadratic map in two complex variables, J. Differential Equations 169 (2001), no. 1, 57-141.

[12] S. Lefschetz, Differential Equations: Geometric Theory, Interscience publishers, 1957.

[13] N. Mehidi and N. Mohdeb, Homoclinic solutions in a quadratic differential system under discretization, J. Difference Equ. Appl. 19 (2013), no. 4, 538-542.

[14] Y. K. Zou and W. J. Beyn, On manifolds of connecting orbits in discretizations of dynamical systems, Nonlinear Anal. 52 (2003), no. 5, 1499-1520.

Noureddine MEHIDI

DÉPARTEMENT DE MATHÉMATIQUES

Laboratoire de Mathématiques Appliquées

Université A. Mira de BÉJaia 06000, Algérie

E-mail address: manogha@yahoo.fr 
NAdia Mohdeb

DÉPARTEMENT DE MATHÉMATIQUES

Laboratoire de Mathématiques Appliquées

Université A. Mira de BéJaia 06000, Algérie

E-mail address: n_mohdeb@hotmail.com 